\title{
Artelogie
}

Recherche sur les arts, le patrimoine et la littérature de l'Amérique latine

5 | 2013

Femmes créatrices en Amérique latine : le défi de synthétiser sans singulariser

\section{Éditorial : Femmes créatrices en Amérique Latine : le défi de synthétiser sans singulariser}

Ana Paula Cavalcanti Simioni, Deborah Dorotinsky et Maira de Luca

\section{OpenEdition}

Journals

Édition électronique

URL : https://journals.openedition.org/artelogie/5102

DOI : 10.4000/artelogie.5102

ISSN : 2115-6395

Éditeur

Association ESCAL

Référence électronique

Ana Paula Cavalcanti Simioni, Deborah Dorotinsky et Maira de Luca, «Éditorial : Femmes créatrices en Amérique Latine : le défi de synthétiser sans singulariser », Artelogie [En ligne], 5 | 2013, mis en ligne le 16 octobre 2013, consulté le 20 décembre 2021. URL : http://journals.openedition.org/artelogie/5102 ; DOI : https://doi.org/10.4000/artelogie.5102

Ce document a été généré automatiquement le 20 décembre 2021.

Association ESCAL 


\title{
Éditorial : Femmes créatrices en Amérique Latine : le défi de synthétiser sans singulariser
}

\author{
Ana Paula Cavalcanti Simioni, Deborah Dorotinsky et Maira de Luca
}

1 Entre 1974 et 1979, dans le sillage du mouvement féministe, l'artiste américaine Judy Chicago a conçu son œuvre emblématique, Dinner Party, en rassemblant dans une fête symbolique mille trente-huit femmes de différentes périodes historiques. Parmi elles, trente-neuf étaient représentées à table, et les autres, nommées sur des céramiques disposées dans une grande installation. Il s'agissait de célébrer, tout en la promouvant, une tradition féminine jusqu'alors méconnue par l'historiographie dominante. Cependant, dans cette grande communion, on pouvait remarquer certaines des limites ethnocentriques du féminisme anglo-saxon : comme l'a observé Estelle Chacon, aucune des héroïnes du « Nouveau Monde » ne figurait parmi les invitées d'honneur à table. Si les « Hispaniques » de même que les « Noires » étaient nommées, elles ne l'étaient que sur quelques tuiles posées à terre, marquant ainsi le lieu symbolique qu'elles occupaient dans ce nouveau récit proposé par le féminisme né aux États-Unis et en Angleterre $^{1}$.

2 Les réactions à l'œuvre de Judy Chicago, devenue une icône du féminisme "d'égalité », ne tarderont pas à arriver. En 1977, Griselda Pollock publia «What's wrong with the Images of Women", dénonçant ce qu'elle appelait une "iconographie vaginale », présente dans l'œuvre, qui tendrait à réduire les multiples identités féminines à un supposé signifiant stable dérivé de leurs corps biologiques. S'opposant à une image univoque, Griselda Pollock - qui est certainement le nom le plus influent aujourd'hui dans l'historiographie de l'art féministe - a proposé la déconstruction des images féminines, la critique des discours stabilisateurs, c'est-à-dire de tous les types de réduction, dont ceux effectués par le féminisme. Depuis les années 1980, d'une manière générale, on peut dire que le féminisme "d'égalité » a cédé la place à celui de "la différence ». Il est important de citer la contribution de Gayatri Spivak Chakavorty à la littérature postcoloniale. Dans «Imperialism and Sexual Difference» (1986) en 
particulier, l'auteure souligne la nécessité de passer d'un féminisme simplement "oppositionnel» à un féminisme "critique ", qui soit en mesure d'examiner continuellement la façon dont le féminisme est lui-même imbriqué dans les institutions et les idéologies. Dans ce sens, des auteurs comme Chela Sandoval (1995) ont joué un rôle important pour la problématisation du féminisme anglo-saxon des années 1960-1970, qui présupposait donc un sujet féministe unique, stable, hégémonique, et qui ne traduisait pas les attentes, les désirs et les réalités vécus par «les autres du féminisme ». Ainsi les dynamiques de classe sociale et d'ethnicité ont-elles commencé à être prises en compte dans les questions "féminines".

On peut affirmer que, dans le champ artistique, ce débat a favorisé les analyses portées sur l'importance des images de la femme propagées dans l'art, la presse et le cinéma, espaces fondamentaux pour la promotion et la cristallisation de discours normatifs sociaux - et alors considérés comme des «technologies du genre » (De Lauretis, 1987). Dès lors, plutôt que de promouvoir une image positive de la féminité, l'historiographie féministe de l'art s'est consacrée à la critique de ces représentations (Arruda, 2013).

4 Cependant, en contemplant les études de genre/art en Amérique Latine, nous sommes confrontés à une situation complexe : l'histoire de la pensée féministe ne s'est en effet pas déroulée de la manière rapportée ci-dessus. Les textes du féminisme de «l'égalité » et de celui de «la différence » sont souvent parvenus aux lecteurs simultanément. De plus, les recherches menées se nourrissent des paradigmes élaborés par les théories anglo-saxonnes, marquées par le célèbre essai fondateur de Linda Nochlin. Dans « Why have there been no great women artists?» (1971), l'auteure soutient que le peu de reconnaissance des femmes dans l'histoire de l'art résulte de facteurs sociaux et institutionnels, en particulier l'inégal accès des femmes à la formation artistique. Ce sont sur ces bases établies qu'une historiographie de l'art féministe s'est appuyée.

Cependant, il faut reconnaître que les réalités multiples contenues dans le terme "Amérique Latine » diffèrent largement de celles qui ont donné lieu à l'émergence de ces théories féministes, de sorte qu'elles doivent nécessairement être révisées, adaptées, voire ré-élaborées, lorsqu'elles sont confrontées à d'autres contextes ${ }^{2}$. Il faut également rappeler que ces postulats n'ont pas connu une réception simultanée ou unanime dans les différents pays d'Amérique Latine. Alors qu'au Mexique, ces textes circulaient et étaient influents dès le début des années 1970 dans les discussions universitaires, parvenant également aux institutions artistiques, comme le prouve l'article d'Andrea Giunta publié ici, dans d'autres cas, notamment au Brésil, où la production artistique féminine et féministe est considérable, le texte fondateur de Linda Nochlin n'a aujourd'hui toujours pas été traduit en portugais, ce qui illustre la distribution limitée de cette littérature. Entre ces deux extrêmes, on peut citer le cas de l'Argentine, dont la capitale a été le théâtre de plusieurs expérimentations féministes dans les années 1970. Ces productions contestaires réalisées depuis les marges du système de l'art sont analysées par Maria Laura Rosa dans «El despertar de la conciencia. Impacto de las teorías feministas sobre las artistas de Buenos Aires durante las décadas del' 70 y ' 80 ». Luana Saturnino Tvardovskas, dans « Tramas feministas na arte contemporânea brasileira e argentina », démontre que les productions féministes au Brésil et en Argentine sont organisées de manière différente des pays anglo-saxons, de façon moins institutionnalisée, moins identitaire, qui ne se traduit pas en de grandes expositions collectives ou dans une révision plus acerbe du canon. L'auteure affirme que cela ne retire en rien la force poétique féminine contenue dans de nombreuses 
œuvres présentées. Ces considérations sont utiles pour mesurer la difficulté de construire un cadre théorique et méthodologique permettant de comprendre des productions et des situations historiques très différentes. Cela se reflète également dans les impasses et les limites institutionnelles, encore très présentes et perceptibles dans les tentatives d'« inclure " les œuvres d'artistes femmes latino-américaines dans les grands récits contemporains, tentatives contribuant à modifier les hiérarchies et à retracer les invisibilités, comme l'analyse ici Erin L. McCutcheon dans «Feminism Unfolding: Negotiating In/Visibility of Mexican Feminist Aesthetic Practices within Contemporary Exhibitions ».

6 Ainsi, le présent numéro d'Artelogie a été envisagé comme une contribution au débat sur le croisement entre les études d'art et de genre, en offrant un panorama large et varié de recherches sur les femmes artistes en Amérique Latine, du XIXème siècle aux expressions contemporaines. Le but n'était pas de reproduire une théorie monolithique ni d'élaborer une cartographie exhaustive de la production des femmes artistes dans l'ensemble des pays d'Amérique Latine. Il est présenté au lecteur une sélection d'articles offrant une densité analytique et une dimension verticale, tout en couvrant des expériences, des productions et des poétiques réalisées dans divers pays et sur une période relativement large. Une priorité a été donnée ici aux formes artistiques ayant encore peu fait l'objet de publications (tels que les arts plastiques, la musique et le design), et les pratiques littéraires, qui bénéficient déjà d'une longue tradition, n'ont volontairement pas été abordées.

7 Un premier ensemble d'essais examine comment les dimensions de genre imprègnent les processus de constitution des discours identitaires qui ont surgi au long du $\mathrm{XIX}^{\text {ème }}$ siècle, dans plusieurs pays d'Amérique Latine, au sein de leurs luttes pour une autonomie politique. Ces discours se matérialisent dans - et par-les formes artistiques. Tout comme les romans de fondation (Sommer, 2010), les tableaux constituent de véritables discours visuels à caractère pédagogique, dans lesquels, au moyen d'articulations complexes (et tendues), des dichotomies alors considérées comme apparemment inconciliables - telles que civilisation/barbarie, dominant blanc/ dominé autochtone ou noir, actif/passif, homme/femme - pouvaient être transcendées, apaisées ou domestiquées. Dans ce processus civilisateur conflictuel impliquant le dilemme du métissage, le corps féminin occupe un espace politique, visuel et symbolique. Par exemple, au Chili, c'est ce que montre l'article de Joséphine de La Maza sur les tableaux de Monvoisin, en particulier Elisa Jaramillo de Bravo Bañados, mujer del cacique; ou encore dans la jeune République brésilienne, c'est ce qu'analysent Tatiana Lotierzo et Lilia M. Schwarcz en s'intéressant à la célèbre toile Redenção de Cã de Modesto Broccos.

8 Un second ensemble d'articles aborde, à travers différentes pratiques artistiques comme la musique, la gravure, la peinture et la sculpture, l'entrée des femmes artistes dans ce que l'on pourrait appeler des «modernités périphériques» (Sarlo, 1999). L'insertion des femmes dans les milieux artistiques a eu lieu tout au long du $\mathrm{XIX}^{\text {ème }}$ siècle dans plusieurs pays latino-américains, en particulier le Mexique, le Brésil, l'Argentine et le Chili : ils ont connu des processus d'institutionnalisation des pratiques artistiques fondées sur des modèles étrangers qui ont mené à la création des Académies 
d'art. Leur modèle a été de manière générale l'Académie française postrévolutionnaire, qui n'acceptait pas les femmes comme élèves, mais leur permettait de présenter leur production dans les Salons (Simioni, 2008). Cela signifiait que les femmes n'avaient pas un plein accès à la formation artistique comme leurs collègues hommes, bien que certaines compétences non seulement tolérées étaient encouragées, dans la mesure où elles étaient constitutives des idéaux bourgeois de la féminité diffusés dans ces pays.

Comme le démontre Georgina Gluzaman dans l'article «El trabajo recompensado: mujeres, artes y movimientos femeninos en la Buenos Aires de entresiglos», une situation ambiguë émergeait en Argentine imprégnée des tensions qui découlaient, d'une part, des attentes sociales normatives sur la féminité idéale, et, d'autre part, des conditions concrètes de professionnalisation dont elles jouissaient. Dans « Del caballete al telar. La Academia Nacional de Bellas Artes, las escuelas profesionales y los debates en torno de la formación artística femenina en la Argentina de la primera mitad del siglo XX ", Julia Ariza renforce ce panorama en se concentrant sur les conditions de la formation artistique à laquelle les femmes avaient accès en Argentine durant le processus de modernisation. Gloria Cortez aborde cette problématique à partir du cas d'artistes chiliennes dans «Estéticas da resistencia: las artistas chilenas y la vanguardia femenina (1900-1936) ». Elle étudie les obstacles à la formation rencontrés par les artistes, ainsi que les modalités esthétiques de "transgression» du discours patriarcal dominant qu'elles sont parvenues à réaliser. Dans « Género y cultura visual. Adrienne Macaire-Bacle en la historia del arte argentino », Lia Munilla, Sandra Szir et Georgina Gluzman s'attachent à l'œuvre pionnière de cette artiste graveur du début du XIX ${ }^{\text {ème }}$ siècle, à Buenos Aires, en effectuant une recherche originale dans les sources de l'époque. Ces auteures examinent la production de cette artiste, négligée, qui travailla dans une modalité alors jugée comme «mineure » et essentiellement "masculine». Centrant également leurs analyses sur les interrelations entre genre et modalités artistiques, Dalila Vasconcellos, dans «Du salon à la scène : l'ascension des femmes et

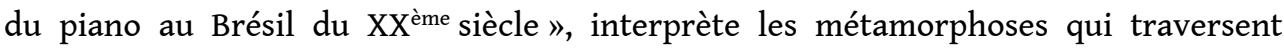
l'univers musical brésilien à la Belle Époque (c.1889-1930), où ont émergé trois pianistes réputées (Antonieta Rudge, Magda Tagliaferro et Guiomar Novaes). L'auteure montre que la condition de pianiste concertiste est progressivement devenue une prérogative féminine, dès lors que les productions et les trajectoires de ces musiciennes ont réussi à dépasser les asymétries traditionnelles de genre. Il en ressort pour elles une position et une assise nouvelles dans le champ musical brésilien socialement légitimé et reconnu.

Nous reprenons ici l'expression «modernités périphériques ", qui permet d'aborder le fait que, dans les régions hispanophones et lusophones, les artistes des avant-gardes modernistes adoptèrent et adaptèrent certaines des formes dérivées des répertoires appelés " ethniques » et/ou " populaires ", développant ainsi un modernisme hybride, ou "anthropophagique $»^{3}$. Dans ce sens, certains articles sont très révélateurs des insertions féminines dans des champs dominés jusque-là par les hommes: l'architecture et le design industriel, des années 1930 aux années 1950 au Mexique et au Brésil. Zeuler R. M. de Lima, dans « Lina Bo Bardi et l'architecture brésilienne de l'après guerre ", expose l'évolution de la carrière et de l'œuvre architecturale de Lina Bo Bardi. Cette architecte italienne, installée à São Paulo à la fin de la Seconde Guerre Mondiale, et qui a réalisé des travaux importants pour le modernisme brésilien, tels que le bâtiment emblématique du MASP (Musée d'Art de São Paulo), est demeurée peu reconnue internationalement, bien que sa réputation n'ait cessé de croître ces dernières années ${ }^{4}$. Ana Elena Mallet aborde quant à elle le parcours de Clara Porset, 
designer industriel d'origine cubaine installée au Mexique. Elle révèle comment ses liens stratégiques avec les architectes reconnus lui ont permis de mettre en avant, à travers des projets de décoration d'intérieur et de mobilier, sa vision de récupération et d'adaptation de formes dérivées du mobilier populaire mexicain. Il est de plus intéressant de noter la contribution théorique fondamentale de Lina Bardi pour la valorisation de l'artisanat brésilien ${ }^{5}$. L'article de Michele Greet traite également du problème de l'articulation entre les élans internationalistes du modernisme et les injonctions locales/nationales/populaires dont les artistes d'Amérique Latine ont fait l'expérience et qu'ils ont mobilisés. L'auteure propose une analyse comparative d'expositions réalisées par quatre artistes latino-américaines à Paris dans les années 1920 : les Brésiliennes Tarsila do Amaral et Anita Malfatti, la Mexicaine Lola Velasquez Cueto et la Cubaine Amelia Pelaez. D'une part, elle analyse comment elles ont chacune établi des stratégies différentes pour s'insérer dans les milieux artistiques parisiens, en opérant des choix concernant les thèmes représentés et la matérialité de leurs œuvres, et, d'autre part, elle montre comment ces décisions ont impliqué des négociations complexes autour du genre de chaque style adopté. Ces articles nous aident à comprendre que la dimension de genre non seulement différencie les créatrices et les modalités, mais implique également la matérialité des pratiques artistiques, ainsi que les degrés de légitimité qui les classent dans leurs champs artistiques.

11 Enfin, nombre d'articles de ce numéro abordent les travaux de femmes artistes dans l'art contemporain, révélant une variété de thématiques les préoccupant et parlant d'elles: le corps, la sexualité, l'histoire des femmes, la violence de genre et la maternitéb. Deux articles analysent le cas du Mexique, qui se présente comme un centre important de production et de réflexion sur les pratiques féministes en Amérique Latine dans les années 1970 : « Cosas de mujeres ? Feminist networks of collaboration in 1970's Mexico» de Gabriela Aceves et «Feminist Disruption in Mexican Art, 1975-1987 » d'Andrea Giunta. L'article de Giunta propose une lecture des interventions réalisées par des artistes - Paula Weiss, Monica Mayer, Magali Lara et la journaliste et activiste féministe Ana Victoria Jiménez - qui ont rendu possible un scénario féministe dans les milieux artistiques mexicains : elles ont introduit des corporalités rebelles et insoumises, qui ont souvent été rendues invisibles par l'historiographie de l'art féministe du pays. L'article contribue ainsi à libérer le potentiel de ces œuvres peu conventionnelles, en intervenant dans un corpus d'images immobilisées par des classifications rigides des discours de l'histoire de l'art.

Le potentiel politique et esthétique transgressif d'artistes femmes est également abordé par une série d'articles qui revisitent des productions réalisées dans les contextes autoritaires, qui se propageaient dans de nombreux pays au cours des années 1960 et 1980. Les œuvres, en tant qu'espaces de résistance politique et d'opposition au discours sur la domesticité féminine "naturelle", sont examinées par Giulia Lamoni dans "(Domestic) Spaces of Resistance: Three Artworks by Anna Maria Maiolino, Letícia Parente and Anna Bella Geiger ", texte centré sur le cas brésilien. Ce contexe historique est repris dans l'article de Gina McDaniel Tarver, consacré à la production de la sculptrice colombienne Feliza Bursztyn ; il est également traité dans l'article de Maria Laura Rose, qui étudie les œuvres de María Luisa Bemberg et de Monique Altschul en montrant le degré de conscience politique et artistique féministe présent dans certains circuits artistiques de la capitale argentine pendant la dictature. L'article de Luana Saturnino aborde la production de deux artistes, une Argentine et une Brésilienne, qui, bien qu'ayant travaillé au cours du processus démocratique dans ces deux pays (dans 
les années 1980 et 1990), sont les héritières d'une poétique féministe qui a éclos et s'est développée dans les décennies précédentes, marquées par un autoritarisme féroce. Il est intéressant de noter que ces poétiques féministes consistent en des «manœuvres radicales $»^{7}$ qui cherchent à contester les discours idéologiques dominants à travers la matérialité des œuvres. En employant notamment la couture, la décoration, les œuvres textiles, la broderie, l'aquarelle, traditionnellement considérées comme artisanales et/ ou domestiques, c'est-à-dire esthétiquement inférieures, ces artistes ont opéré des glissements de sens, les dotant des nouvelles significations, et ont ainsi mis à nu les asymétries et les inégalités sociales inscrites dans les mondes des arts. La subversion, le déplacement et la déconstruction des images de la féminité passe ainsi par la transformation des procédures, des modalités, des pratiques et des hiérarchies artistiques établies.

13 Cet ensemble d'articles offre un panorama complexifié et incisif sur la contribution des artistes femmes à une critique politique. Comme l'affirme Nelly Richard, la condition d'êtres doublement dominés a permis aux artistes de passer de la "marginalité comme externalité du pouvoir à la marge comme questionnement du symbolisme du pouvoir » (Richard, 2011 : p. 40). Il faut toutefois noter que, bien que les œuvres soient acerbes, cela ne signifie pas que les artistes s'identifient comme " féministes " : nombre d'entre elles ont refusé et refusent encore aujourd'hui de se voir cataloguées comme telles. Il convient donc de souligner la différence reconnue, au sein des pratiques artistiques et politiques, entre un désir de militance "générale » et une militance féministe tout court. Nombre des œuvres et des artistes discutées ici signalent des poétiques féministes, sans que leurs auteures se voient attachées à quelque type de militance extérieure à son propre champ artistique. Les articles qui traitent de productions contemporaines témoignent d'une transformation des médias utilisés par les artistes: la photographie, la vidéo, l'installation et la performance, ou encore les techniques mixtes, sont devenues prédominantes. Dans le cas de l'artiste portoricaine, Elsa María Meléndez, abordée par Raquel Arzola, la manipulation de techniques mixtes telles que la sérigraphie, le découpage en broderie et le montage dans des boîtes, sont employées comme éléments majeurs pour proposer une syntaxe des scénarios sociaux de circulation du corps féminin. Nous pouvons également constater l'usage de l'appropriation et de la re-signification de l'imagerie traditionnelle chez une artiste importante, la brésilienne Adriana Varejao. En analysant son œuvre, Alice Heeren met en évidence les croisements entre race/genre et clivages sociaux. Ce type d'interprétation se trouve également dans l'article de Jamie Ratliff sur l'œuvre photographique de la mexicaine Daniela Rosell, "Ricas y famosas », qui examine le thème de l'espace domestique, abordé dans d'autres articles mentionnés précédemment. Ratliff part d'une lecture de l'espace de la «maison » en tant que pierre de touche de la nation mexicaine, un espace qui définit traditionnellement ce que sont les fonctions d'une femme. Dans cette œuvre, la maison apparaît comme un espace de représentation dans lequel le pouvoir et l'autorité de l'État envahissent les vies des citoyens et permettent la reproduction du pouvoir en perpétuant les rôles traditionnels de genre.

15 Enfin, nous tenons à souligner que le dossier comporte également une rubrique intitulée "Reprise», dans laquelle nous souhaitons re-publier des textes classiques considérant l'art et le genre en Amérique Latine, et encore peu accessibles au grand public. Le texte de Laura Malosetti Costa «El rapto de las cautivas blancas : un aspecto 
erótico de la barbarie en la plástica Rioplatense del siglo XIX » est un des exemples pionniers d'interprétation au moyen de la perspective de genre du mythe de la conquête, racontée par les «blancs ", tel qu'il apparait dans la littérature et les arts plastiques. Avec créativité, profondeur et de manière très novatrice, Malosetti entrecroise dans cet article classique les sources littéraires et les sources visuelles, énonçant la soustraction des femmes blanches par les Indiens (scènes d'enlèvement et de captivité), avec ce qui signale une jonction entre les problèmes de genre, de race et de classe sociale. Par ailleurs, l'article de Angelica Velazquez, "Juliana and Josefa Sanromán: The Representation of Bourgeois Domesticity in Mexico, 1850-1860» présente la production plastique des sœurs Sanromán, élèves du directeur de la chaire de peinture à l'Académie de San Carlos, en montrant comment ces jeunes filles mexicaines ont pu accéder à la formation de peintre et quelles étaient leurs thématiques de prédilection, par rapport à la classe sociale à laquelle elles appartenaient. En sus de ces articles, un entretien est proposé avec l'historienne de l'art argentine Laura Malosetti Costa, dont les réflexions sur les relations entre art, genre et pouvoir, présentes dans ses nombreuses publications, conférences, etc., promettent de stimuler la réflexion et la recherche dans d'autres contextes socio-politiques.

Les textes réunis ici nous permettent de mesurer l'ampleur des réactions face aux constructions canoniques des genres, "les guerres" contre ces canons comme le mentionne Gloria Cortez, puisque de nos jours il est impossible de contourner la productivité critique et rhétorique des contre-discours (et contre-histoires) que les stéréotypes de genre, de race et de classe ont généré dans les pays d'Amérique Latine.

\section{BIBLIOGRAPHIE}

ARRUDA, Lina Alves (2013). Estratégias desconstrutivas: a crítica feminista da representação". Dissertação de Mestrado em Artes Visuais, Escola de Comunicações e Artes, São Paulo, 2013. BROUDE, Norma Broudeand and GARRARD, Marry (1982). Feminist Art History: Questioning the Litany.New York, Harper \& Row Publishers.

BARTRA, Eli. (2005) Mujeres en el Arte Popular. De Promesas, traiciones, monstrous y celebridades. México, Universidad Autónoma Metropolitana.

CANCLINI, Nestor G. (1990). Culturashibridas. Estrategias para entrar y salir de lamodernidad, México, Grijalbo.

DE DIEGO, Estrella (2008). "Durante El feminismo de la igualdad: Historiografía, teoría y prácticas artísticas”. Madrid, Exitbook: feminismo y arte de género, n.9.

DE LAURETIS, Teresa. (1987). Technologies of Gender.Essays on Theory, Film and Fiction.Bloominton, Indiana University Press.

DOROTINSKY, Deborah (2008). “Mirar desde los márgenes o los márgenes de la mirada. Fotografía por dos mujeres indígenas de Chiapas”, Debate Feminista, Año 19,Vol. 38, octubre 2008. 
EDER, Rita. (1982). “Las mujeres artistas en México”, en Anales del Instituto de Investigaciones Estéticas, México, IIE-UNAM, Vol XIII, núm. 50, tomo 2, pp.251-259.

FERRAZ, Isa Grinspum (org.)(1994). Tempos de grossura. O design no impasse. Série Pontos sobre o Brasil. São Paulo, Instituto Lina Bo e P.M. Bardi.

GIASSON, Patrice (ed.) (2010). Brincando fronteras. Creaciones locales mexicanas y globalización. México, CONACULTA.

GIUNTA, Andrea (2011). "Estrategias de lamodernidad en América Latina”. In: Escribir las Imágenes.Buenos Aires, SigloVeintiuno.

HOLANDA, Heloisa e HERKENHOFF, Paulo (2006).Manobras radicais. Rio de Janeiro, Centro Cultural Banco do Brasil.

HUYSSEN, Andrea (2010). “Geografias Del Modernismo”, In: Modernismo Después de la Posmodernidad.Buenos Aires, Gedisa Editorial.

JONES, Amelia (2011). “ Les politique ssexuelles de The Dinner Party. Un contexte très critique (1996-2005), IN: DUMONT, Fabienne (ed). La Rébellion du Deuxième Sexe. L'histoire de l'art au crible des théories féministes anglo-américaines (1970-2000). Les Presses du Reél.

MAYAYO, Patricia. (2008). '¿Por qué no ha habido (grandes) artistas feministas en España?. Apuntes sobre una historia en busca de autor' en Xabier Arakistain; Lourdes Méndez:Producción artística y teoríadel arte: nuevos debates I, Vitoria, Centro Cultural MontehermosoKulturunea.

NOCHLIN, Linda (1991).Why there be no great women artists?". In:_Art and Sexual Politics. New York, Macmillan Publishing Co.

POLLOCK, Griselda.(1999) “Tracing figures of presence, naming ciphers of abscence. Feminism, Imperialism, and Post modernity in the Work of SutapaBiswas". In BLOOM, Lisa. (1999) With other eyes. Looking at Race and Gender in Visual Culture.Minneapolis / Londres, University of Minnesota Press.

POLLOCK, Griselda (1977). “What's wrong with Images of Women?”, Screen Education, 1977, no24, 25-33.

RUBINO, Silvana (2009). Lina por escrito: textos escolhidos de Lina Bo Bardi, 1943-1991. São Paulo, Cosac\&Naify.

SANDOVAL, Chéla Sandova (1995).l "Feminist forms of Agency and Oppositional Consciousness: U.S. Third World Femenist Criticism" en KEGAN GARDINER, Judith. (1995) Provoking Agents.Genderand Agency in Theoryand Practice. Urbana and Chicago, Universityof Illinois Press. SARLO, Beatriz (1999). Una Modernidad Periférica: Buenos Aires 1920-1930. Buenos Aires, Nueva Vision. SCHWARTZ, Jorge (2003). Brasil: 1920-1950. Da Antropofagia à Brasília.São Paulo: Museu de Arte Brasileira/ Cosac \&Naify.

SIMIONI, Ana Paula C. (2008). Profissão artista: pintoras e escultoras acadêmicas brasileiras (1884-1922). São Paulo, EDUSP/FAPESP.

SPIVAK, Gayatri Chakravorty. (1986) “Imperialismand Sexual Difference”.Oxford Literary Review 8, pp.517-529.

SOMMER, Doris. (2010)“Um romance irresistible: las ficciones fundacionales de América Latina”. In: BHABHA, Homi (compilador). Nación y Narración. Buenos Aires, SigloVeintiuno Editores. 


\section{NOTES}

1. Au sujet de cette œuvre, voir le lien suivant : https://www.brooklynmuseum.org/exhibitions/ dinner_party/

2. Il est intéressant de noter que certaines auteures espagnoles ont établi des critiques très similaires à la nécessité de repenser l'hégémonie des modèles anglo-saxons pour expliquer la production artistique de leur pays; à ce sujet voir De Diego (2008), Mayayo (2008). Pour davantage d'investigations sur ce thème, voir les articles de Luana Saturnino et de Maria Laura Rosa dans le présent dossier.

3. Dans la vaste bibliographie sur cette question, notons entre autres Canclini (1990), Sarlo (1999), Schwartz (2003), Huyssen (2010), Giunta (2011).

4. Rubino (2009).

5. À ce sujet voir « Tempo de Grossura », publié initialement en 1980 et organisé par Isa Grispum Ferraz (1994).

6. L'équipe éditoriale reconnait que le travail des artistes d'origine indigène n'a pas été contemplé ici comme il le mérite. À ce sujet le lecteur peut consulter Bartra (2005), Dorotinsky (2008) et Giasson (2010), entre autres.

7. Holanda et Herkenhoff (2006).

\section{AUTEURS}

ANA PAULA CAVALCANTI SIMIONI

IEB/USP

DEBORAH DOROTINSKY

UNAM

MAIRA DE LUCA

CRAL-EHESS 